\title{
Nuclear Transporting Factor 2 as a Novel Biomarker of Head and Neck Squamous Cell Carcinoma and Associated with T/B Cell Receptor Signaling Pathway
}

\author{
Tingmin Zhang $\mathbb{D}^{\mathbb{D}}$, Yue Xi $\mathbb{D}$, Tianfu Wu $\mathbb{D}$, and Jianfeng Liu $\mathbb{1}$ \\ The State Key Laboratory Breeding Base of Basic Science of Stomatology (Hubei-MOST) \& Key Laboratory of Oral Biomedicine \\ Ministry of Education, School \& Hospital of Stomatology, Wuhan University, 430079 Wuhan, China
}

Correspondence should be addressed to Tianfu Wu; wutianfu@whu.edu.cn and Jianfeng Liu; liujianfeng@whu.edu.cn

Received 26 July 2021; Accepted 6 January 2022; Published 4 February 2022

Academic Editor: Shibiao Wan

Copyright (C) 2022 Tingmin Zhang et al. This is an open access article distributed under the Creative Commons Attribution License, which permits unrestricted use, distribution, and reproduction in any medium, provided the original work is properly cited.

\begin{abstract}
Objective. This study is aimed at exploring the role of nuclear transporting factor 2 (NUTF2) in head and neck squamous cell carcinoma (HNSCC) based on The Cancer Genome Atlas (TCGA) database. Methods. We obtained 528 HNSCC patients' clinical data from TCGA and performed expression level analysis of NUTF2. Gene Sets Enrichment Analysis (GSEA) was conducted to identify NUTF2-associated regulatory mechanisms in HNSCC. In addition, several other tools were used to enrich the regulatory network. Results. We found that NUTF2 was significantly upregulated $(P<0.001)$ in HNSCC. We then observed that higher NUTF2 is associated with poorer overall survival and disease-free survival. Further, by using Cox analyses, we determined high NUTF2 as an independent risk factor of predicting poorer overall survival. Tumor immune infiltration analysis revealed a significantly negative correlation between NUTF2 expression and the level of tumor infiltrated CD8 ${ }^{+} \mathrm{T}$ cell and B cell, suggesting that NUTF2 may be involved in the immune regulation of HNSCC. Gene sets related to T/B cell receptor signaling pathways were differentially enriched based on the NUTF2 expression phenotype. KEGG pathways were used to show that NUTF2 may affect proliferation, differentiation, and immune response of T/B cell through regulating PI3K/AKT, NF $\kappa$ B, MAPK, and Calcium signaling pathways. Conclusion. NUTF2 might be a valuable biomarker for HNSCC and correlated with T/B cell receptor signaling pathway.
\end{abstract}

\section{Introduction}

Head and neck cancers (HNSC) occur in the mucosal linings of the aerodigestive tract, including the lip, oral cavity, salivary glands, pharynx, and larynx. About 900,000 new cases of HNSCC occurred, and over 400,000 patients died from it per year $[1,2]$. Head and neck squamous cell carcinomas (HNSCC) account for $90 \%$ of HNSC [3]. The prognosis for patients with HNSCC is unfavorable and largely determined by the cancer stage and TNM classification. Typically, the survival rate of patients decreases rapidly with cancer progression $[4,5]$. For HPV-positive HNSCC, early viral screening can be used to diagnose high-risk groups. At the same time, the high expression of PD-L1 in HPV-positive HNSCC provides evidence for the feasibility of immunotherapy [6].
Vaccine and drug advances continue to be made, making the prognosis for these patients far better than that for the HPV-negative ones [7]. So far, no evidence-based screening protocols have been validated for all patients with HNSCC [8]. Traditional treatment options for HNSCC including surgical and nonsurgical methods have reached a bottleneck in improving patient survival and prognosis [9]. The advent of immunotherapy is the most remarkable advance in the field of HNSCC treatment in the past decade. For patients with refractory and/or metastatic (R/M) HNSCC, immunotherapy provides an option to maintain long-term remission while other therapies have limited effect [10]. Clinical trials have shown that immunotherapy can be used to enhance the effectiveness of conventional therapies, and this combination therapy will be used in the first line [11]. Limited 
immunotherapeutic targets were found to be effective, and the immune response to single antigen targeted therapy is at a poor level. Synergy trials are underway, and molecular targeted drugs will be combined with immunologic agents in later stages [10].

NUTF2 (nuclear transporting factor 2, also known as NTF2) is a factor that mediates the import of GDP-bound Ran from the cytoplasm into the nucleus through nuclear pore complexes (NPCs). In previous studies, NUTF2 was the cargo that imported the small GTPase Ran [12]. The process that Ran-GDP bound to NPCs was mediated by NUTF2, which showed that NUTF2 played an essential role in the translocation stage of nuclear protein import $[13,14]$. Other results found that the level of NUTF2 could regulate the efficiency of protein transport between nucleus and cytoplasm [15]. In previous studies of nuclear transporters, they were often associated with tumor genesis. In particular, cargo and NPCs play a regulatory role in altered nuclearcytoplasmic transport patterns in cancer cells [16]. In 2016, Stelma et al. examined the Karyopherin superfamily, a kind of transport receptor, involved in the regulation of cancer through alterations in gene expression [17]. The potential of nuclear transporters as biomarkers and therapeutic targets was highlighted. However, few studies have explored the relationship between NUTF2 and cancers. An experiment in Xenopus laevis cells found that decreased level of NUTF2 may contribute to melanoma development through the regulation of cell nuclear size [18]. On the other hand, in 2020, $\mathrm{Du}$ et al. found that NUTF2 is a downstream target of LINC00173 and regulates tumorigenesis in glioma; its overexpression awakens the proliferation, migration, and invasion of cells [19]. The association between NUTF2 and HNSCC has not been illustrated in previous studies.

In this study, we demonstrated NUTF2 expression in HNSCC and investigated its clinical significance. The correlations between NUTF2 expression and tumor infiltrated $\mathrm{CD}^{+} \mathrm{T}$ cell and $\mathrm{B}$ cell were confirmed. Through using GSEA, we explored the mechanism that may be related to NUTF2 in the regulation of tumor immunity.

\section{Materials and Methods}

2.1. Clinical Data from TCGA. 528 HNSCC patients' clinical characteristics including age, gender, status, grade, and different cancer classifications were downloaded from TCGA (https://portal.gdc.cancer.gov/). Furthermore, because of the relevance between the NUTF2 expression and clinical message, we obtained the gene expressions of these patients from TCGA. To support further research like CNV analysis, the copy number variation data were also downloaded from TCGA. Before the analysis, incomplete data had been deleted. Detailed information is shown in Table 1.

2.2. Statistical Analysis. Statistical analysis was performed by the $\mathrm{R}$ software (version 3.6.1 (2019-07-05)). The Wilcoxon rank-sum test was used to compare the expression of NUTF2 in the patient's normal tissues and cancer tissues. In the clinical correlation analyses, the Wilcoxon rank-sum test was used when only two groups of samples were com-
TABLE 1: Patient characteristics in TCGA-HNSCC.

\begin{tabular}{|c|c|c|}
\hline Clinical characteristics & Total 528 & $\%$ \\
\hline \multicolumn{3}{|l|}{ Age } \\
\hline \multicolumn{3}{|l|}{61 (average) } \\
\hline \multicolumn{3}{|l|}{$(19-90)$} \\
\hline \multicolumn{3}{|l|}{ Gender } \\
\hline Male & 142 & 26.9 \\
\hline Female & 386 & 73.1 \\
\hline Not available & 1 & 0.2 \\
\hline \multicolumn{3}{|l|}{ Status } \\
\hline Alive & 329 & 62.3 \\
\hline Deceased & 199 & 37.7 \\
\hline \multicolumn{3}{|l|}{ Grade } \\
\hline G1 & 63 & 11.9 \\
\hline $\mathrm{G} 2$ & 311 & 58.9 \\
\hline G3 & 125 & 23.7 \\
\hline G4 & 7 & 1.3 \\
\hline GX & 18 & 3.4 \\
\hline Not available & 4 & 0.8 \\
\hline \multicolumn{3}{|l|}{ Stage } \\
\hline Stage I & 27 & 5.1 \\
\hline Stage II & 74 & 14.0 \\
\hline Stage III & 82 & 15.5 \\
\hline Stage IV & 270 & 51.1 \\
\hline Not available & 75 & 14.2 \\
\hline \multicolumn{3}{|l|}{$\mathrm{T}$ classification } \\
\hline T0 & 1 & 0.2 \\
\hline $\mathrm{T} 1$ & 49 & 9.3 \\
\hline $\mathrm{T} 2$ & 140 & 26.5 \\
\hline T3 & 101 & 19.1 \\
\hline $\mathrm{T} 4$ & 175 & 33.1 \\
\hline $\mathrm{TX}$ & 39 & 7.4 \\
\hline Not available & 23 & 4.4 \\
\hline \multicolumn{3}{|l|}{ M classification } \\
\hline M0 & 191 & 36.2 \\
\hline M1 & 1 & 0.2 \\
\hline MX & 65 & 12.3 \\
\hline Not available & 271 & 51.3 \\
\hline \multicolumn{3}{|l|}{$\mathrm{N}$ classification } \\
\hline No & 180 & 34.1 \\
\hline N1 & 68 & 12.9 \\
\hline $\mathrm{N} 2$ & 72 & 13.6 \\
\hline N3 & 8 & 1.5 \\
\hline NX & 75 & 14.2 \\
\hline Not available & 25 & 4.7 \\
\hline
\end{tabular}

pared, and the Kruskal-Wallis rank-sum test was used when the sample groups were more than two, reaching three or more. Moreover, cox regression analysis was used to determine NUTF2 as a risk factor for HNSCC by using the Survival package of $\mathrm{R}$. Besides, the expression data with 
$\mid \log 2$ fold change (FC)| was adopted, which made HR more pronounced than raw data. The amendatory $P$ values were also used to show the significance of HR. The data of NUTF2 copy number variations (CNVs) in two kinds of tissues was checked by the chi-square test, and the adjusted $P$ value was adopted. The Circos 2D track plot was produced by the RCircos package of R [20].

2.3. GEPIA. Gene Expression Profiling Interactive Analysis (GEPIA, version 2, http://gepia2.cancer-pku.cn/\#index) was a visualized gene expression analytical tool. In this study, NUTF2 expression compared in different subtypes of HNSCC was based on TCGA data from GEPIA, and the boxplot was downloaded from GEPIA. The survival Kaplan-Meier curve was plotted on GEPIA as well.

2.4. Gene Set Enrichment Analysis (GSEA). Gene Set Enrichment Analysis (GSEA, version 3.0, http://software.broadinstitute.org/ gsea/index.jsp) is a genomewide expression analytical tool that detects different sets of genes between two biological groups [21]. In this study, GSEA was performed to reveal biological pathways enriched in high-NUTF2 groups. The NUTF2 expression level was used as a phenotype label. The number of gene set permutations was 1,000 for each analysis. Annotated gene sets c2.cp.kegg.v7.0.symbols.gmt were chosen to be the reference gene sets. The nominal $P$ value $<0.05$ and false discovery rate $(\mathrm{FDR})<0.25$ were set as the cut-off criteria.

2.5. TIMER. Tumor Immune Estimation Resource (TIMER, version 2.0, http://timer.cistrome.org/) is an enhanced version developed recently based on the original TIMER, which is a web server for comprehensive tumor-immune interactions research [22]. TIMER 2.0 integrates multiple algorithms for immune infiltration estimation and allows users to explore various associations between immune infiltrates and genetic features in the TCGA cohorts [23]. Our results were using the Microenvironment Cell Populationscounter (MCP-counter) method, which allows the robust quantification of the absolute abundance of eight immune and two stromal cell populations in heterogeneous tissues from transcriptomic data [24].

2.6. Connectivity Map. Connectivity Map (cMap, version 2.0, also known as L1000, https://clue.io) is an online platform that connects genes, drugs, and disease states by gene expression signatures [25]. This new version of cMap contains 1.3 million L1000 profiles, and its analytical tools allow researchers to discover the mechanism of action of small molecules, functionally annotate genetic variants of disease genes, and inform clinical trials.

\section{Results}

3.1. Patient Characteristics in TCGA-HNSCC. As shown in Table 1, the clinical data downloaded from TCGA database consists of 528 HNSCC patients. The median age at diagnosis was about 61 years old. $26.9 \%$ patients were male, and $73.1 \%$ were female. The living status included $329(62.3 \%)$ dead and 199 (37.7\%) alive. Histological grade I could be found in 63 (11.9\%) patients, G2 in 311 (58.9\%), G3 in 125 $(23.7 \%)$, G4 in $7(1.3 \%)$, and GX in $18(3.4 \%)$. In tumor classification, $1(0.2 \%)$ in all was T0, $49(9.3 \%)$ were T1, 140 (26.5\%) were T2, $101(19.1 \%)$ were T3, 175 (33.1\%) were $\mathrm{T} 4$, and 39 (7.4\%) were TX. In metastasis classification, most patients $(n=366,63.6 \%)$ were not sure whether their cancer cells metastasized, 191 (36.2\%) were M0, and only $1(0.2 \%)$ was M1. And in regional lymph node classification, node involvement was not observed in $180(34.1 \%)$ patients, 68 (12.9\%) were N1, $72(13.6 \%)$ were $\mathrm{N} 2,8(1.5 \%)$ were $\mathrm{N} 3$, and $75(14.2 \%)$ were NX.

3.2. Upregulated NUTF2 Expression in HNSCC. Compared with the adjacent normal tissues, the results indicated that NUTF2 expression was significantly upregulated in the tumor tissues (Figure 1(a)). Furthermore, the paired analysis, in which normal and tumor tissue come from same patient, showed a significant upregulation of NUTF2 expression in HNSCC (Figure 1(b)). GEPIA database was used to check NUTF2 expression in different subtypes of HNSCC. With four major subtypes of HNSCC, atypical, basal, classical, and mesenchymal, NUTF2 expression in three subtypes was significantly higher in cancer tissues compared with adjacent normal tissues $(P<0.01)$; only the atypical subtype showed no significant difference, but high NUTF2 expression in tumor tissue was still observed in the boxplot (Figure 1(c)). To probe the mechanism of NUTF2 amplification in HNSCC, we examined the copy number variation (CNV) of this gene in patients with HNSCC. A positive correlation between copy number variation and NUTF2 expression was found (Figure 2(a)). Besides, the increased copy number of NUTF2 in HNSCC patients can be observed from the Circos 2D track plot (Figure 2(b)). We concluded that NUTF2 expression was significantly upregulated in HNSCC, which related to the increase of CNV.

3.3. Diagnostic and Prognostic Value of NUTF2 Expression in HNSCC. To explore the clinical significance of NUTF2, we compared NUTF2 expression in HNSCC patients according to different clinical features. However, there was no significant difference between different groups in age, TN classification, histological grade, and cancer stage (Figure 3).

To assess the prognostic value of NUTF2 for HNSCC, patients were divided into two groups, one with low NUTF2 expression and the other with high NUTF2 expression. We found significant differences between the two groups both in overall survival (OS, Figure 4(a)) and disease-free survival (DFS, Figure 4(b)). The survival rate (both OS $(P<0.05)$ and DFS $(P<0.001))$ in high NUTF2 group was lower than that in low NUTF2 group. This result indicated that high NUTF2 expression had a positive association with low survival rate of HNSCC patients. Hazard ratio (HR) implied that high NUTF2 expression might have a predictive value for lower survival rates in HNSCC patients (OS: $\mathrm{HR}=1.7, P<0.05$; DFS: $H R=1.7, P<0.001)$. To check NUTF2 as a risk factor for HNSCC, the univariate and multivariate cox regression analysis was performed. Through univariate analysis, we confirmed that expression of NUTF2 had a close connection with OS in HNSCC ( $\mathrm{HR}=1.465,95 \% \mathrm{CI}=1.037-2.070$, 


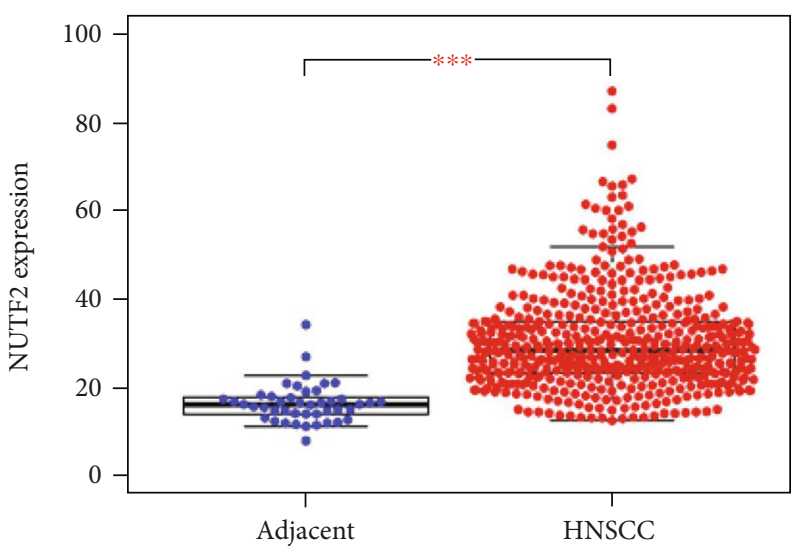

(a)

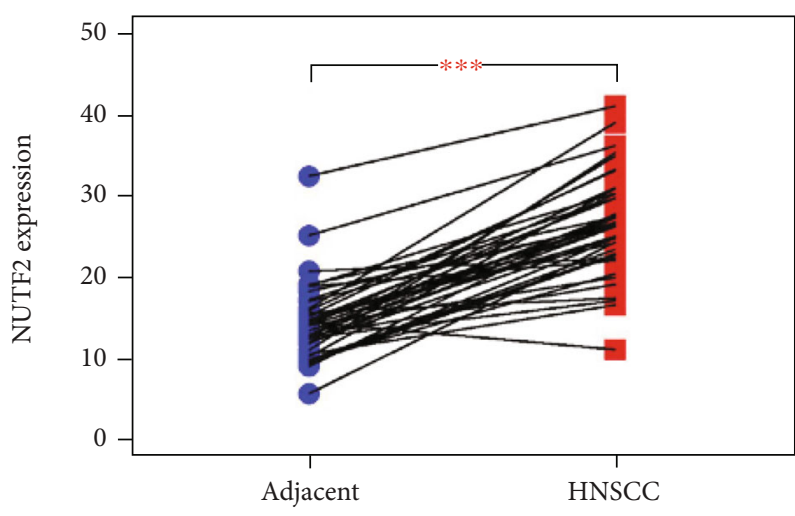

(b)

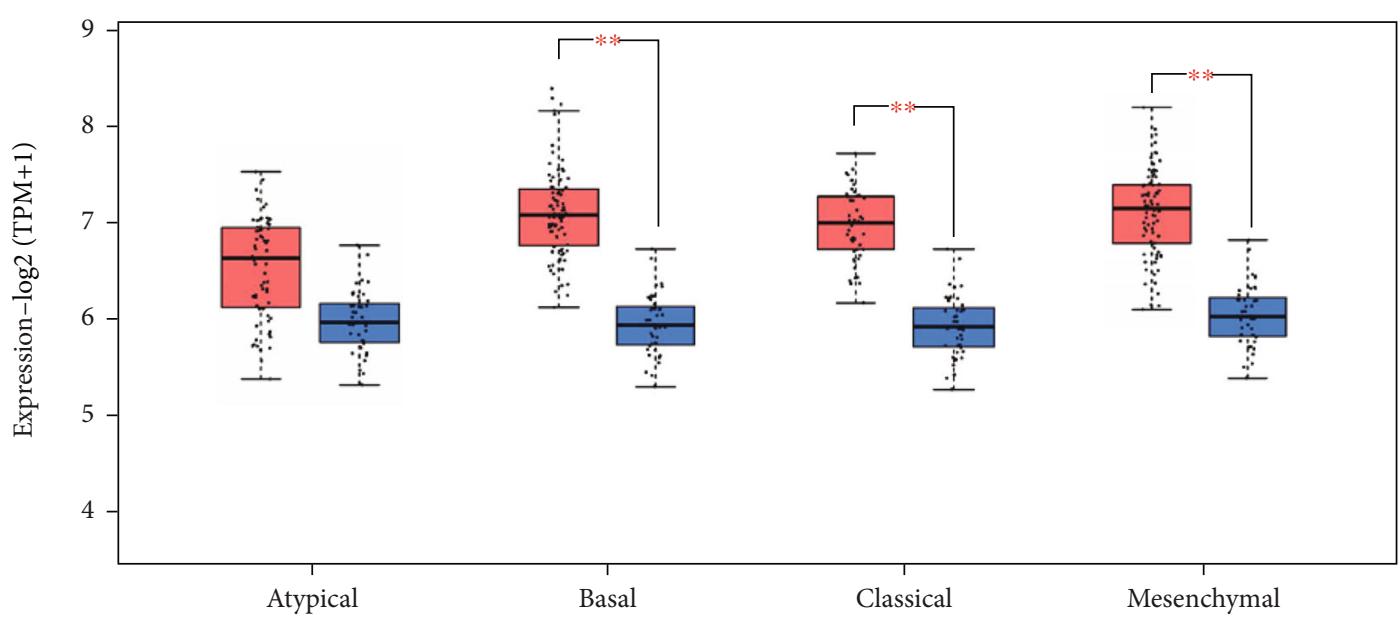

(c)

FIGURE 1: NUTF2 expression in the tumor tissues vs. the adjacent normal tissues. (a) NUTF2 showed significantly higher expression in the tumor tissues than in the adjacent normal tissues; (b) NUTF2 was significantly upregulated in HNSCC compared with the paired noncancerous adjacent tissues from same patient using Wilcoxon rank-sum test; (c) NUTF2 was upregulated significantly in basal, classical, and mesenchymal subtypes of HNSCC. The red box (on the left) represents tumor, and the blue box (on the right) represents normal. Statistical significance compared with the control is indicated by ${ }^{*} P<0.05,{ }^{* *} P<0.01$, and ${ }^{* * *} P<0.001$.

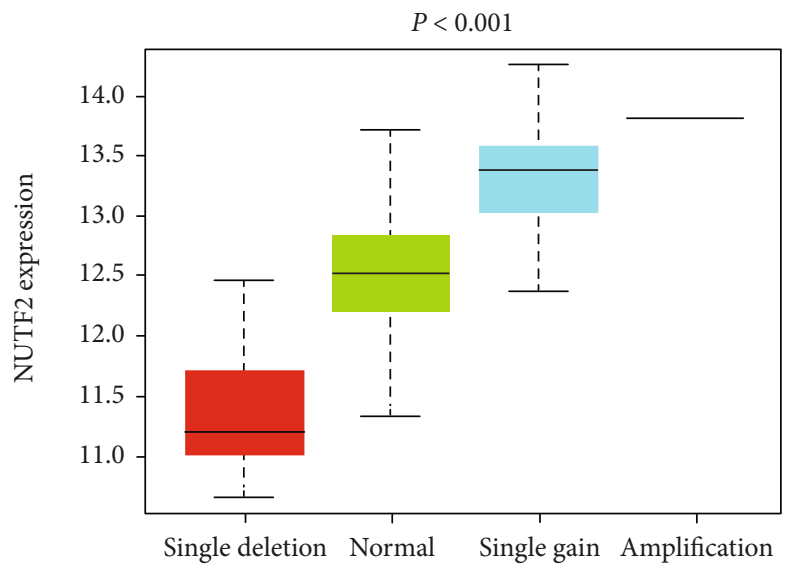

(a)

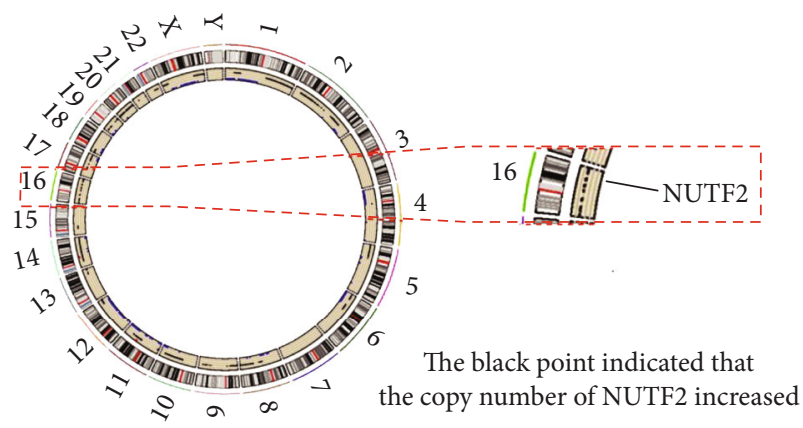

(b)

Figure 2: CNV analysis of NUTF2 in HNSCC. (a) Positive correlation between NUTF2 copy number and NUTF2 expression; (b) NUTF2 was located on chromosome 16. The black point at the corresponding site indicated that the copy number of NUTF2 in cancer tissues usually increased. 


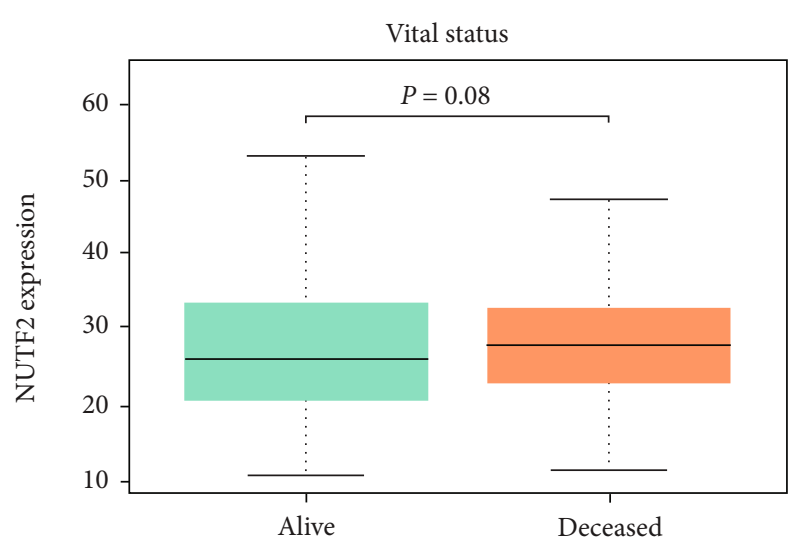

(a)

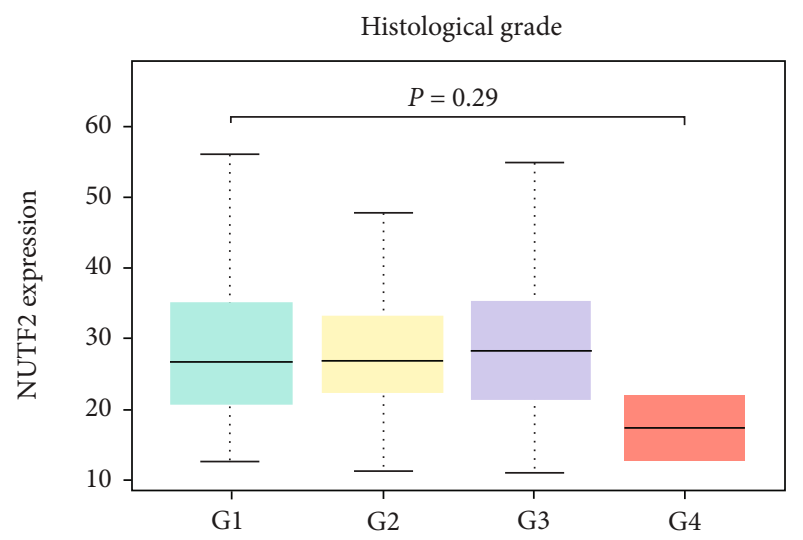

(c)

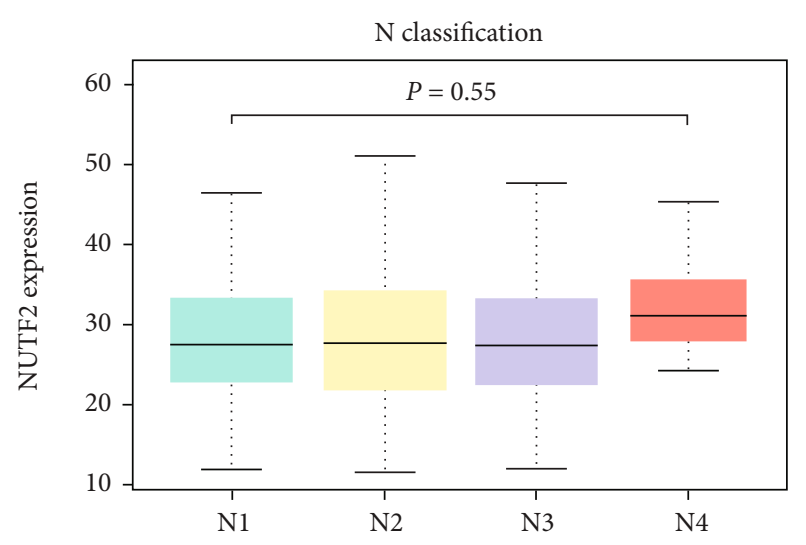

(e)

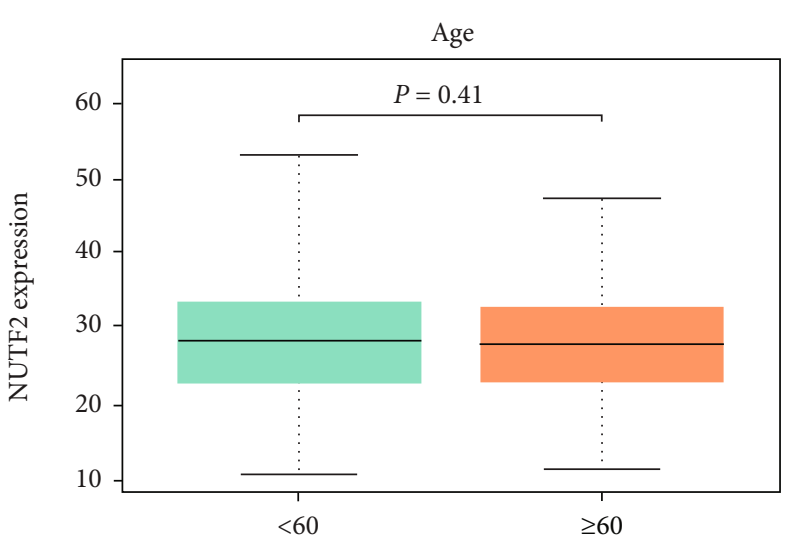

(b)

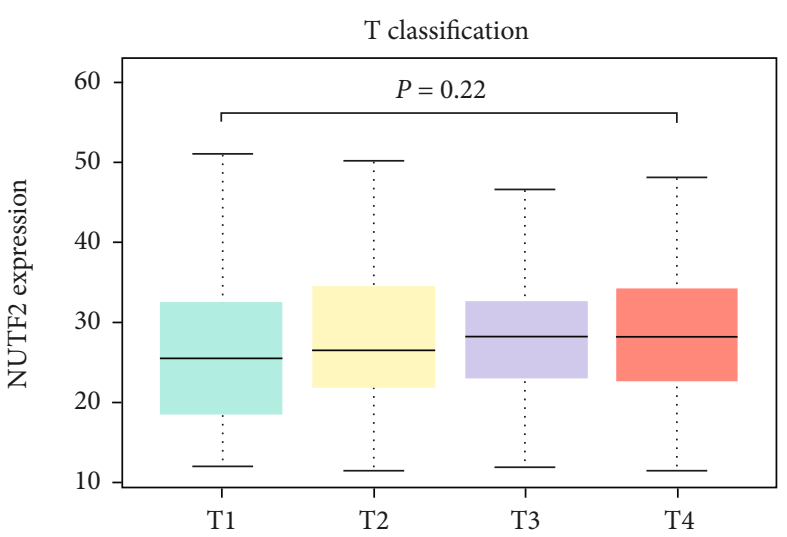

(d)

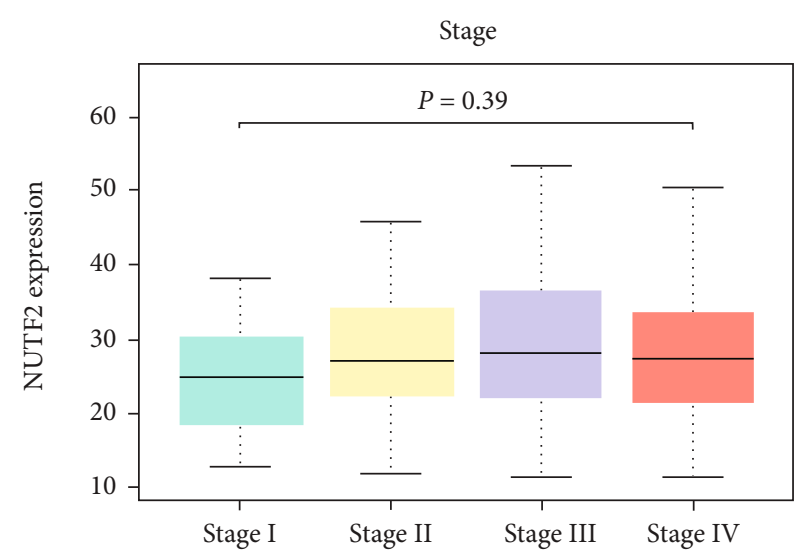

(f)

FIGURE 3: Comparison of NUTF2 expression in HNSCC according to vital status (a), age (b), histological grade (c), T classification (d), N classification (e), and stage (f).

$P<0.05)$. In multivariate analysis with relevant clinical feature adjustment, such as age, gender, grade, stage, T classification, and $\mathrm{N}$ classification, the result shown in Table 2 further confirmed that high NUTF2 expression was an independent risk factor associated with significantly worse overall survival $(\mathrm{HR}=1.456,95 \% \mathrm{CI}=1.020-2.079, P<0.05)$.

3.4. Tumor Immune Infiltration Analysis by TIMER. To uncover the relationship between NUTF2 expression and tumor immune infiltration, Tumor Immune Estimation
Resource (TIMER) was used. As the figure showed, the population of tumor infiltrated $\mathrm{CD}^{+} \mathrm{T}$ cell and B cell in HNSCC had significantly negative correlations with NUTF2 expression (Figures 5(b) and 5(c), Rho $<0$, $\mid$ Rho $\mid>0.3, P<0.05$ ). While the tumor purity of HNSCC showed no relationship with NUTF2 expression, which excluded the interference of NUTF2 on the tumor microenvironment (Figure 5(a)). With tumor purity unregulated, the correlations further illustrated the possible role of NUTF2 in the regulation of tumor infiltrated $\mathrm{CD}^{+} \mathrm{T}$ cell and $\mathrm{B}$ cell. 


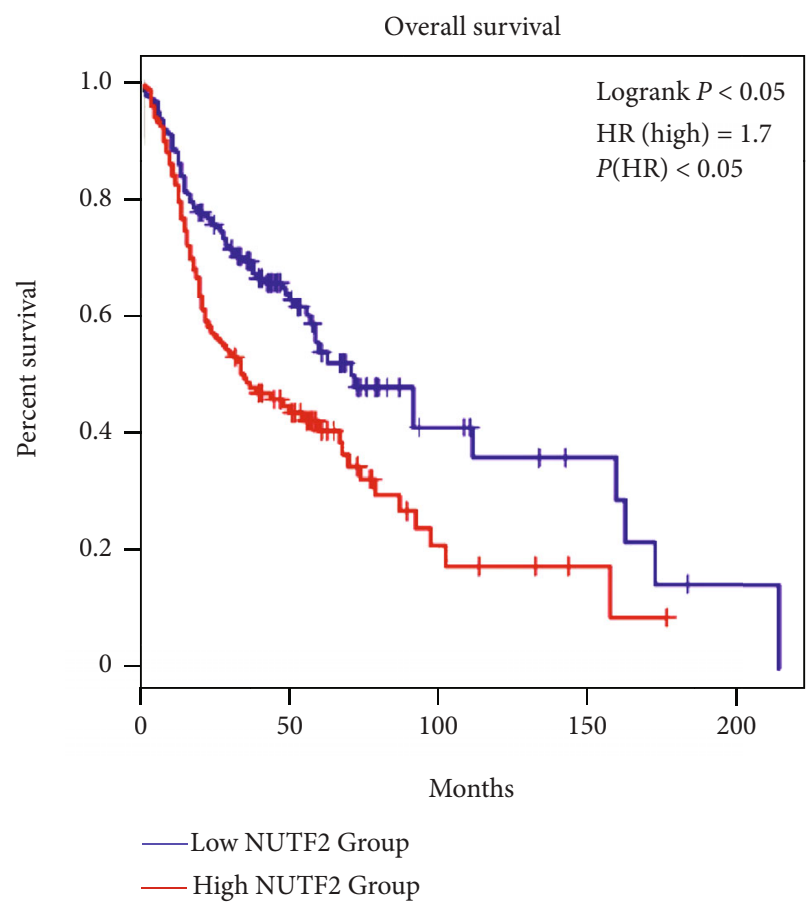

(a)

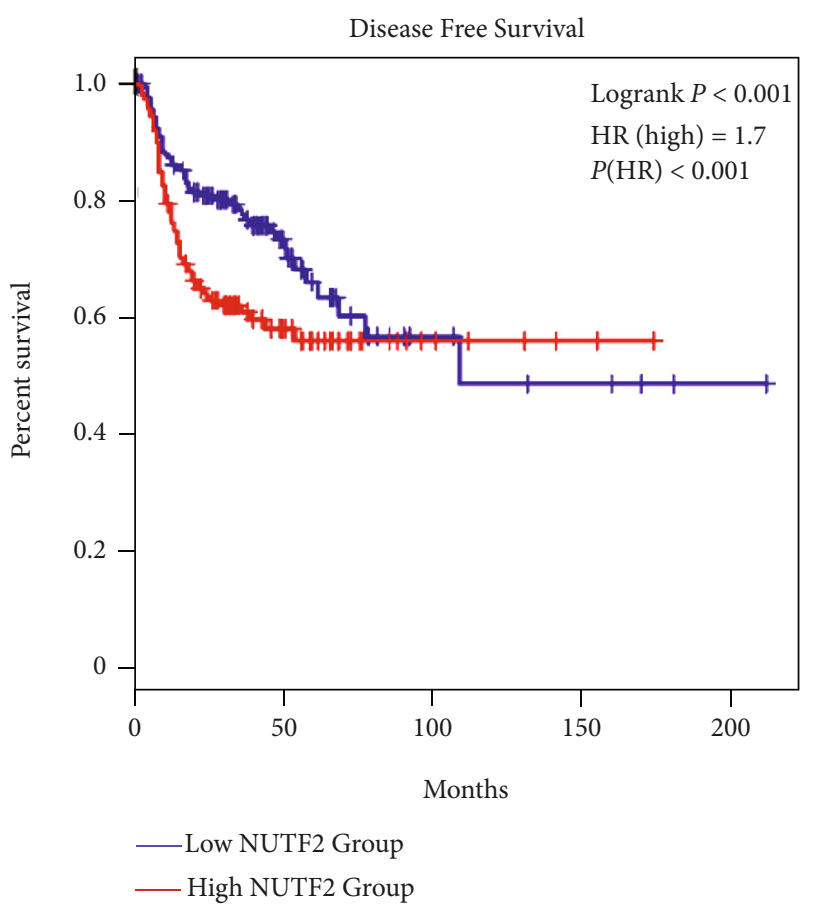

(b)

FIGURE 4: Survival analysis of NUTF2 based on data from TCGA-HNSCC in GEPIA database. The results showed significant correlations between higher NUTF2 expression with poorer overall survival ((a), $P<0.05)$ and Disease Free Survival $((\mathrm{b}), P<0.001)$. HR: hazard ratio.

TABLE 2: Univariate and multivariate cox analysis of overall survival in patients with HNSCC.

\begin{tabular}{lccccc}
\hline \multirow{2}{*}{ Parameter } & HR & Univariate analysis & \multicolumn{3}{c}{ Multivariate analysis } \\
& $95 \%$ CI & $P$ value & HR & 1.025 & $1.008-1.043$ \\
\hline Age & 1.020 & $1.004-1.036$ & 0.014 & 0.798 & $0.550-1.158$ \\
Gender & 0.723 & $0.506-1.035$ & 0.076 & 1.026 & $0.774-1.360$ \\
Grade & 1.177 & $0.903-1.533$ & 0.228 & 1.327 & $0.887-1.986$ \\
Stage & 1.627 & $1.279-2.069$ & $\leq 0.001$ & 1.028 & $0.795-1.330$ \\
T & 1.316 & $1.104-1.570$ & 0.002 & 1.375 & $1.088-1.739$ \\
N & 1.527 & $1.273-1.832$ & $\leq 0.001$ & 1.456 & 0.857 \\
NUTF2 & 1.465 & $1.037-2.070$ & 0.030 & 0.168 \\
\hline
\end{tabular}

HR: hazard ratio; CI: confidence interval.

3.5. Identification of Potential Pathways by GSEA. To identify the altered pathways related to NUTF2 in tumor immunity of HNSCC, Gene Set Enrichment Analysis (GSEA) was conducted between low and high NUTF2 expression groups of HNSCC cases. As Figures 6(a) and 6(b) showed, gene sets related to $T$ cell receptor signaling pathway and $B$ cell receptor signaling pathway were differentially enriched with the low NUTF2 expression phenotype, which suggested NUTF2 negatively regulated $\mathrm{T}$ cell receptor signaling pathway and $\mathrm{B}$ cell receptor signaling pathway. The relevant genes of $\mathrm{T}$ cell receptor signaling pathway and $\mathrm{B}$ cell receptor signaling pathway were shown in Figures 6(c) and 6(d). Among them, the core genes of the leading edge subsets which were most closely associated with $\mathrm{T} / \mathrm{B}$ cell receptor pathway enrich- ment and the specific pathways they regulate were shown in Figure 6(e). Furthermore, we exhibit the proteins encoded by the enriched core genes in the signaling pathways, and the results demonstrated the negative correlation between these proteins and NUTF2 expression. The proteins encoded by the core enrichment genes were concentrated in PI3K/ Akt, NF $\kappa$ B, MAPK, and Calcium signaling pathways, which determined the proliferation, differentiation, anergy, and immune response of T/B cells (Figure 7). This result suggests that the negative role of NUTF2 in the regulation of T/B cells through the above pathways.

3.6. Candidate Small Molecule Drugs Based on cMap. To identify potential small molecule drugs targeting NUTF2, 


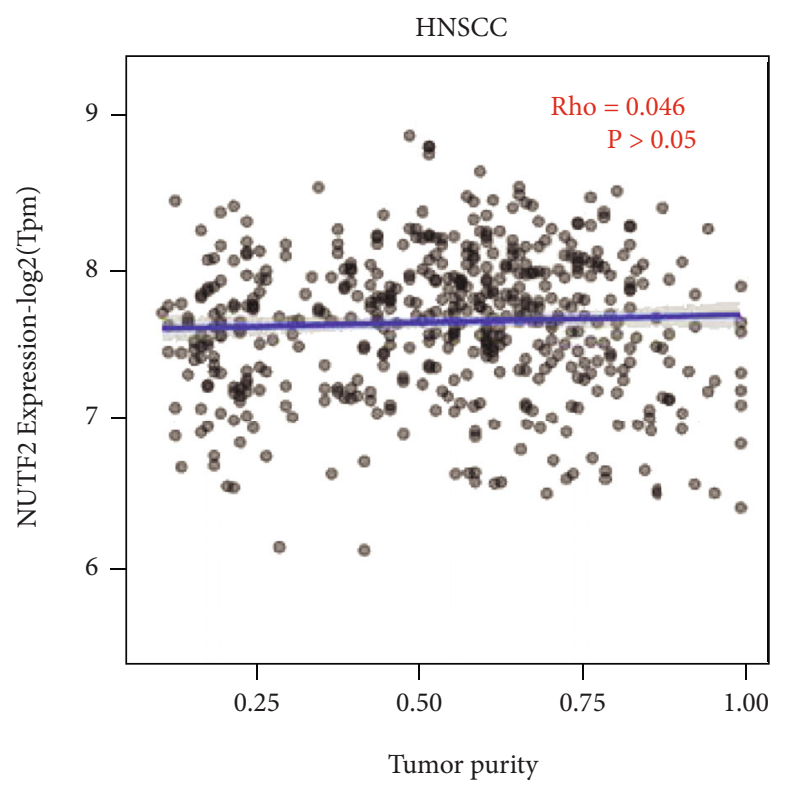

(a)

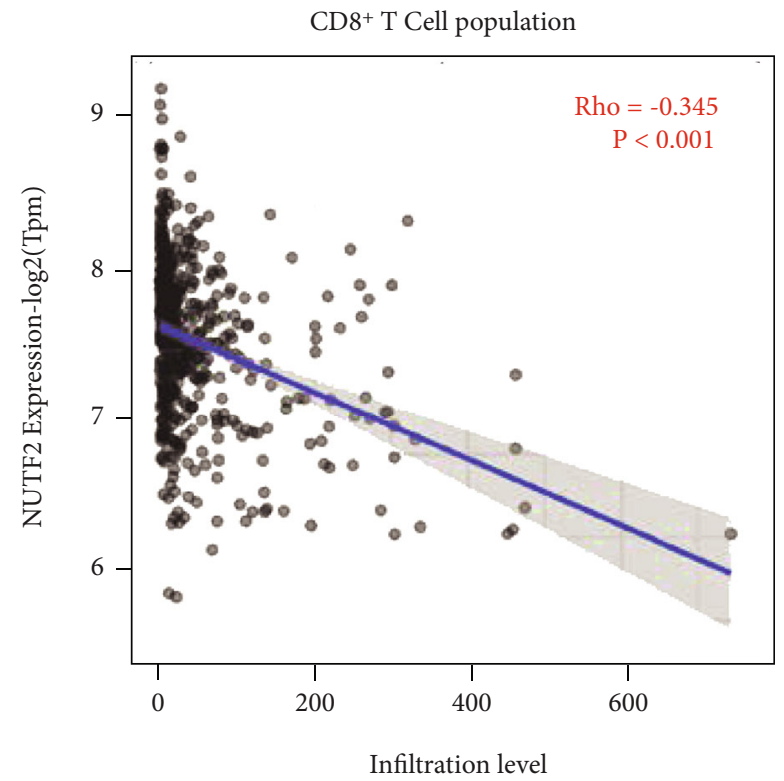

(b)

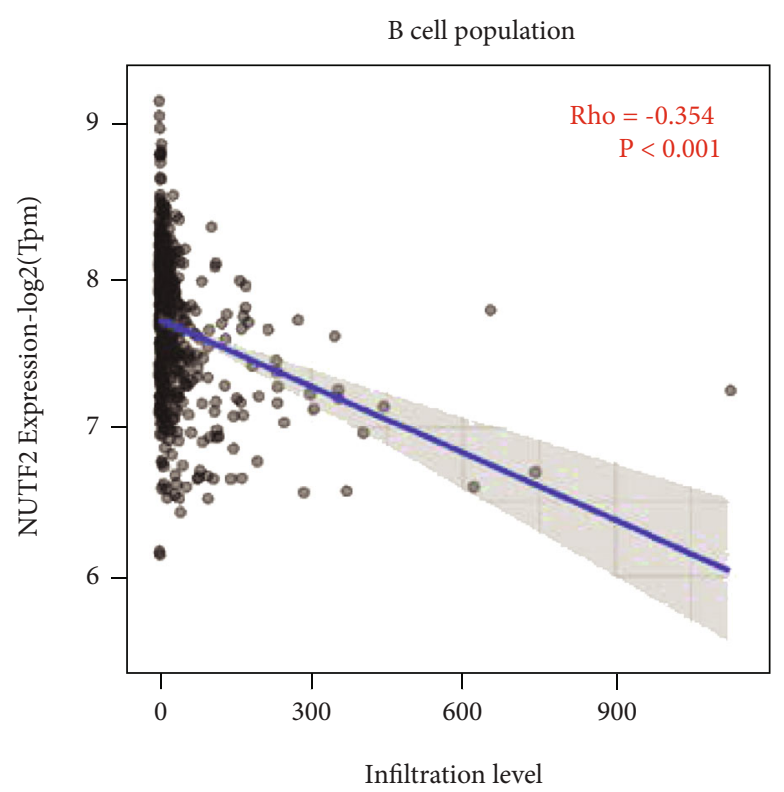

(c)

FIGURE 5: The relationship between NUTF2 expression and immune cell infiltration level. The relationship between tumor purity of HNSCC and NUTF2 expression (a) was shown on left as a control. CD8+ T cell (b) and B cell (c) infiltration levels had negative correlations with NUTF2 expression (Rho $<0$, $\mid$ Rho $\mid>0.3, P<0.001$ ).

Connectivity Map (cMap) was performed to screen out candidate molecule compounds. 9 genes expressed similarly with NUTF2 in HNSCC were detected by GEPIA; then, the 10 upregulated genes were uploaded to Connectivity Map. The output contained the name of compounds, their corresponding connectivity score, target, and mode of action. The connectivity score ranked from -100 to 100 . The closer it gets to -100 , the more likely it would be the potential drug for HNSCC. The 10 most significant compounds were benzbromarone, mosapride, ITE, azathioprine, orphenadrine, H-9, pergolide, protriptyline, CITCO, and rhamnetin (Table 3 ).

\section{Discussion}

Previous studies have demonstrated nuclear transporters regulate carcinogenesis by nuclear transporting in cancer cells. However, few studies have explored the topic of NUTF2 with cancer. This study is aimed at demonstrating the role of NUTF2 in HNSCC.

We found a significant upregulation of NUTF2 expression in HNSCC patients, and high NUTF2 expression was associated with poor overall survival. The same result was observed by Huang's research [26]. Besides, we found higher NUTF2 expression in different HNSCC subtypes as well as 


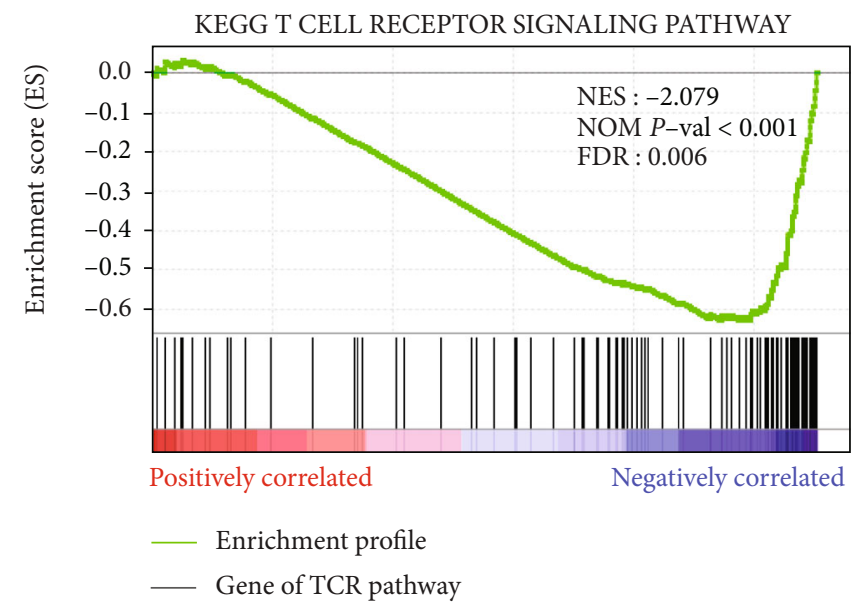

(a)

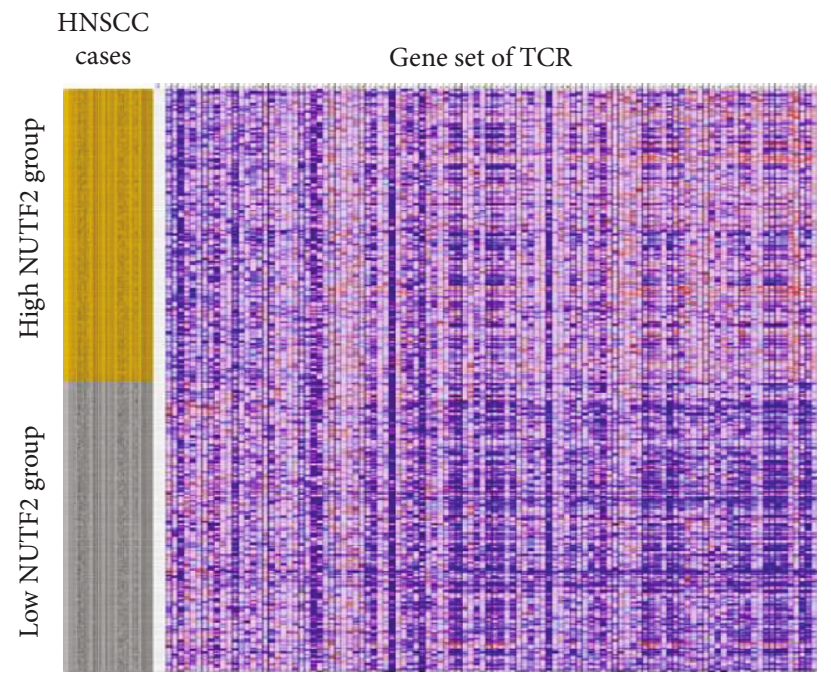

(c)

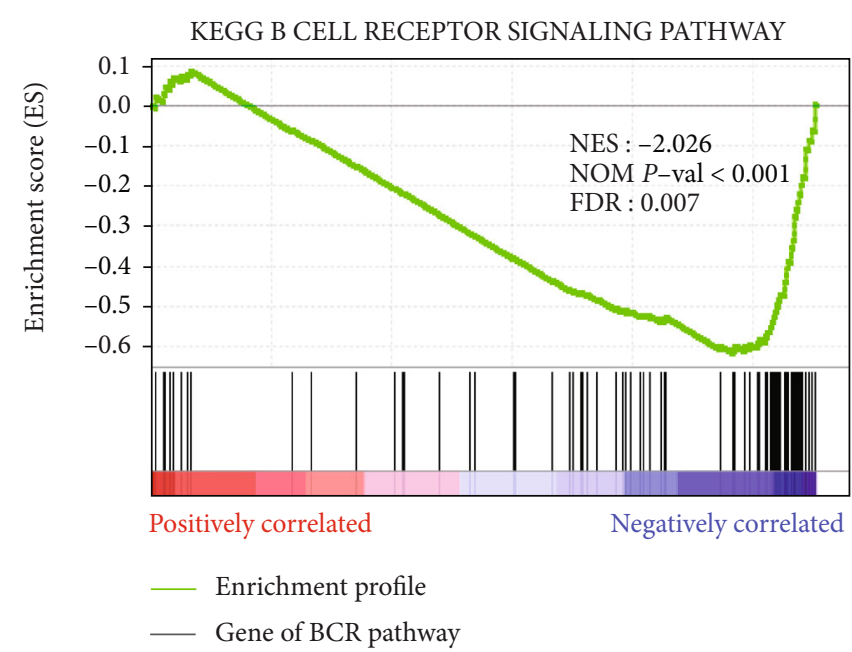

(b)

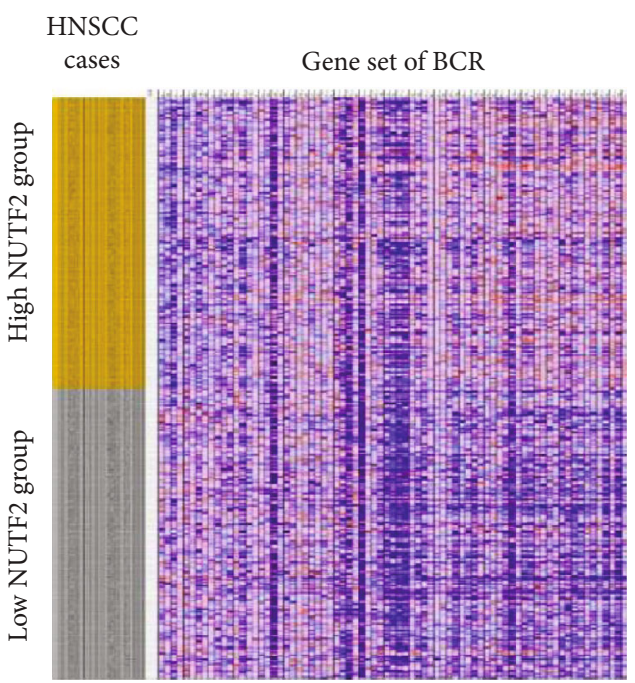

(d)

\begin{tabular}{|ll|}
\hline Enriched pathway & Core genes \\
\hline P13k-AKT signaling pathway & PIK3CA, PIK3CB, PIK3CG, PIK3R1, PIK3R3, PIK3R5, IKBKB, AKT2, AKT3 \\
\hline NF kB signaling pathway & IKBKG, NFKB1 \\
\hline MAPK signaling pathway & RAF1, KRAS, MAPK1 \\
\hline Calcium signaling pathway & NFATC1, NFATC2, NFATC3, NFAT5 \\
\hline
\end{tabular}

(e)

FIGURE 6: Enrichment plots from GSEA. Gene sets related to T cell receptor signaling pathway (a, c) and B cell receptor signaling pathway (b, d) were differentially enriched in HNSCC cases with low NUTF2 expression $(P<0.001)$. The table illustrated the top 4 pathways enriched among the leading edge subsets. TCR: T cell receptor; BCR: B cell receptor.

in paired comparisons of tumor tissue with normal tissue from the same patients. Moreover, we discussed the prognostic value of NUTF2 in HNSCC by DFS analysis, which excludes patients who survive but relapse with HNSCC, offering patients a direct clinical benefit on tumor recurrence and can be a more valuable survival index. Furthermore, univariate and multivariate Cox analyses confirmed NUTF2 expression as an independent risk factor of HNSCC. Based on the results above, NUTF2 is a useful prognostic biomarker of HNSCC.
Early reports showed that NUTF2 engaged in protecting from diabetic retinopathy through depressing vascular endothelial growth factor (VEGF) expression [27]. Moreover, NUTF2 aggregation may alter nucleoplasm transport in nerve cells of Alzheimer's disease patients, leading to neuronal abnormalities and death [28]. RB NLS-mediated nuclear import was critically regulated by NUTF2 [29]. In addition, it was observed that NUTF2 cell localization was different between young cells and senescent cells, suggesting that NUTF2 may influence the cell aging process to some extent 


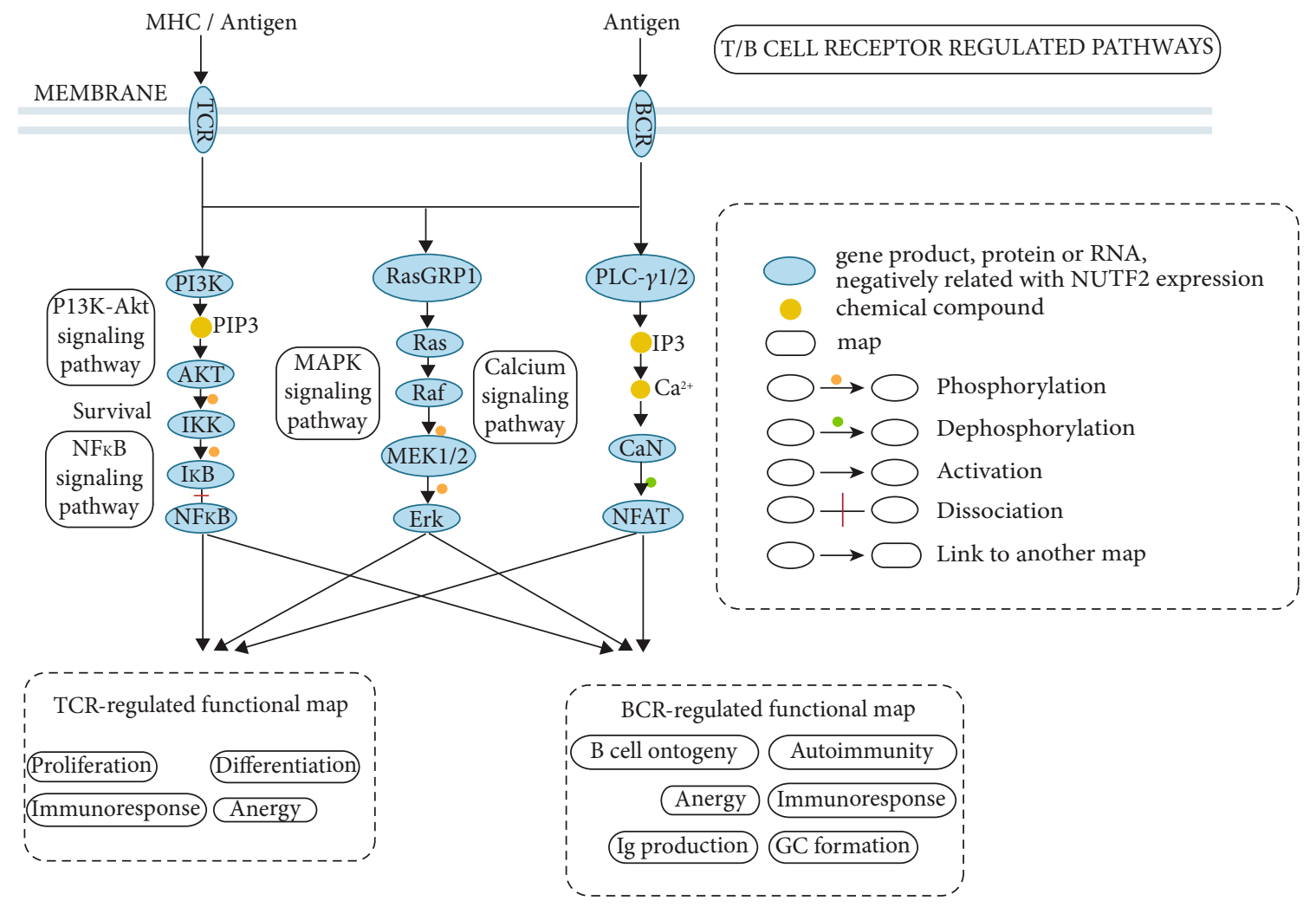

FIgURE 7: Protein regulation in T/B cell receptor signaling pathways. Core enrichment genes analyzed by GSEA were enriched in four signaling pathways including $\mathrm{PI} 3 \mathrm{~K} / \mathrm{Akt}, \mathrm{NF} \kappa \mathrm{B}, \mathrm{MAPK}$, and Calcium signaling pathways. TCR and BCR regulate their respective functional modules through these four pathways. TCR: T cell receptor; BCR: B cell receptor.

TABLE 3: Top 10 most significant compounds based on cMap analysis.

\begin{tabular}{lccc}
\hline cMap name & Score & Target & MOA \\
\hline Benzbromarone & -99.93 & ABCC1, SLC22A12 & Chloride channel blocker \\
Mosapride & -99.89 & HTR4, HTR3A & Serotonin receptor agonist \\
ITE & -99.89 & AHR & Aryl hydrocarbon receptor agonist \\
Azathioprine & -99.89 & HPRT1, IMPDH1, PPAT & Dehydrogenase inhibitor \\
Orphenadrine & -99.86 & CYP2B6, GRIN1, GRIN2D, GRIN3A, GRIN3B, HRH1, SCN10A, SLC6A2 & Acetylcholine receptor antagonist \\
H-9 & -99.86 & PRKACA & PKA inhibitor \\
Pergolide & -99.86 & HTR1A, HTR1B, HTR1D, HTR2A, HTR2B, HTR2C, ADRA1A, & Dopamine receptor agonist \\
& ADRA1B, ADRA1D, KCNA5 & Tricyclic antidepressant \\
Protriptyline & -99.82 & SLC6A2, SLC6A4 & CAR agonist \\
CITCO & -99.82 & NR1I3 & HDAC inhibitor \\
Rhamnetin & -99.79 & ALOX5, MAPK8 &
\end{tabular}

MOA: mode of action.

[30]. In tumor research, long noncoding RNA (lncRNA) LINC00173 could promote the development of disease by enhancing NUTF2 expression in glioma [19]. Besides, the level of NUTF2 expression was downregulated and negatively correlated with nuclear size in melanoma tissue [18]. NUTF2 overexpression was examined to exhibit melanoma metastasis, cell proliferation, and increase apoptosis. Increasing NUTF2 levels plays a negative role in the progression of melanoma. In addition, NUTF2 was expected to pro- vide a target for combination therapy as a novel treatment for cancers; in prostate cancer cells, it improved drug sensitivity and reduced cell proliferation; in melanoma, it downregulated factors that caused drug resistance [31, 32].

In this study, the tumor immune infiltration analysis suggested negative correlations between NUTF2 expression and infiltrated T cell and B cell levels in HNSCC. Moreover, negative correlations were found between NUTF2 expression and $\mathrm{T}$ cell receptor signaling pathway and $\mathrm{B}$ cell 
receptor signaling pathway. As we know, through antigen presentation, $\mathrm{T}$ cells and $\mathrm{B}$ cells were activated and suppressed the tumor progression [33], and the dysfunction of $\mathrm{T}$ cells and $\mathrm{B}$ cells leads to the immune escape of tumor cells [34]. Many tumor cells inhibit the effector function of tumor-specific T cells, inducing a state of anergy [35]. T/B cell costimulator is known to activate downstream signaling molecules including $\mathrm{NF} \kappa \mathrm{B}, \mathrm{MAPK}, \mathrm{ErK}$, and nuclear factor of activated T cells (NFAT) [36-38]. The aberrant expression of NUTF2 in T/B cells resulted in the disorder of $\mathrm{PI} 3 \mathrm{~K} / \mathrm{Akt}, \mathrm{NF} \kappa \mathrm{B}, \mathrm{MAPK}$, and Calcium signaling pathways, which determine the immune response and fate of $T / B$ cells. Our findings suggested that NUTF2 may participate in the immune regulation through regulating $\mathrm{T} / \mathrm{B}$ cell receptor signaling pathways in the process of tumor oncology in HNSCC.

Besides, cMap analysis was used to search for candidate small molecule drugs. 10 upregulated genes (NUTF2 and other 9 genes detected by GEPIA) in HNSCC were submitted, and the output showed several compounds that have a high negative correlation and potential to treat HNSCC. Among the top 10 compounds, some have been proven to have anticancer effects, such as ITE. ITE is an AhR (aryl hydrocarbon receptor) ligand; the AhR can inhibit cellular proliferation in a ligand-dependent manner and act as a tumor suppressor. In previous studies, ITE was examined to be able to suppress the proliferation and migration of several kinds of cancer cells, such as breast, endogenous, and ovarian cancer cells [39-41]. Moreover, ITE was found to regulate $\mathrm{T}$ cell function in inflammation and autoimmune diseases [42, 43]. In addition, pergolide may play an indirect immunomodulatory role by regulating the level of adrenocorticotropic hormone [44]. Therefore, we consider the identified molecule drugs might have the potential to treat HNSCC.

HNSCC is an immunosuppressive type of cancer [45]. Clinical trials have demonstrated that chemotherapy combined with immunotherapy can improve overall survival in patients with HNSCC partly [40]. We found that NUTF2 was associated with the regulation of $\mathrm{T} / \mathrm{B}$ cell receptor signaling pathways; therefore, it may be a potential target for treatment of HNSCC. In summary, our work showed the role of NUTF2 in HNSCC and explored several possible pathways; however, the specific functions and regulatory mechanism still need further research.

\section{Data Availability}

The clinical data used to support the findings of this study were obtained from The Cancer Genome Atlas (TCGA) database (https://portal.gdc.cancer.gov/).

\section{Conflicts of Interest}

The authors declared that they have no conflicts of interest to this work.

\section{Authors' Contributions}

Tingmin Zhang and Yue Xi contributed equally to this work.

\section{Acknowledgments}

This research was supported by the Health Commission of Hubei Province scientific research project (No. WJ2021M179) and the Students' innovation project of school of medicine of Wuhan University (No. MS2019042).

\section{References}

[1] F. Bray, J. Ferlay, I. Soerjomataram, R. L. Siegel, L. A. Torre, and A. Jemal, "Global cancer statistics 2018: GLOBOCAN estimates of incidence and mortality worldwide for 36 cancers in 185 countries," CA: A Cancer Journal for Clinicians, vol. 68, no. 6, pp. 394-424, 2018.

[2] R. L. Siegel, K. D. Miller, and A. Jemal, "Cancer Statistics, 2020," CA: A Cancer Journal for Clinicians, vol. 70, no. 1, pp. 7-30, 2020.

[3] M. P. Curado and M. Hashibe, "Recent changes in the epidemiology of head and neck cancer," Current Opinion in Oncology, vol. 21, no. 3, pp. 194-200, 2009.

[4] K. K. Ang, J. Harris, R. Wheeler et al., "Human papillomavirus and survival of patients with oropharyngeal cancer," The New England Journal of Medicine, vol. 363, no. 1, pp. 24-35, 2010.

[5] W. C. Cho, "Nasopharyngeal carcinoma: molecular biomarker discovery and progress," Molecular Cancer, vol. 6, no. 1, 2007.

[6] K. Przybylski, E. Majchrzak, L. Weselik, and W. Golusiński, "Immunotherapy of head and neck squamous cell carcinoma (HNSCC). Immune checkpoint blockade," Otolaryngologia polska $=$ The Polish otolaryngology, vol. 72, no. 6, pp. 10-16, 2018.

[7] S. N. Whang, "Recent progress in therapeutic treatments and screening strategies for the prevention and treatment of HPV-associated head and neck cancer," Viruses, vol. 7, no. 9, pp. 5040-5065, 2015.

[8] N. Cohen, "Epidemiology and demographics of the head and neck cancer population," Oral and Maxillofacial Surgery Clinics of North America, vol. 30, no. 4, pp. 381-395, 2018.

[9] M. Canning, G. Guo, M. Yu et al., "Heterogeneity of the head and neck squamous cell carcinoma immune landscape and its impact on immunotherapy," Developmental Biology, vol. 7, 2019.

[10] J. D. Cramer and B. Burtness, "The changing therapeutic landscape of head and neck cancer," Nature Reviews. Clinical Oncology, vol. 16, no. 11, pp. 669-683, 2019.

[11] H. F. Kao and P. J. Lou, "Immune checkpoint inhibitors for head and neck squamous cell carcinoma: current landscape and future directions," Head \& Neck, vol. 41, Supplement 1, pp. 4-18, 2019.

[12] I. Cushman, B. R. Bowman, M. E. Sowa, and O. Lichtarge, "Computational and biochemical identification of a nuclear pore complex binding site on the nuclear transport carrier NTF2," Journal of Molecular Biology, vol. 344, no. 2, pp. 303-310, 2004.

[13] W. D. Clarkson, A. H. Corbett, B. M. Paschal et al., "Nuclear protein import is decreased by engineered mutants of nuclear transport factor 2 (NTF2) that do not bind GDPRan"," Journal of Molecular Biology, vol. 272, no. 5, pp. 716-730, 1997.

[14] K. Ribbeck, G. Lipowsky, and H. M. Kent, "Ntf2 mediates nuclear import of ran," The EMBO Journal, vol. 17, no. 22, pp. 6587-6598, 1998. 
[15] T. Tachibana and M. Hieda, "exogenously injected nuclear import factor p10/ntf2 inhibits signal-mediated nuclear import and export of proteins in living cells," FEBS Letters, vol. 397, no. 2-3, pp. 177-182, 1996.

[16] T. R. Kau, "Nuclear transport and cancer: from mechanism to intervention," Nature Reviews. Cancer, vol. 4, no. 2, pp. 106117, 2004.

[17] T. Stelma, A. Chi, P. J. Van Der Watt, and A. Verrico, “Targeting nuclear transporters in cancer: diagnostic, prognostic and therapeutic potential," IUBMB Life, vol. 68, no. 4, pp. 268$280,2016$.

[18] L. D. Vukovic, B. A. Stohr, and D. L. Levy, “Abstract 475: Ntf2 regulates nuclear size in mammalian cells and may contribute to altered nuclear size in melanoma," Cancer Research, vol. 76, pp. 475-475, 2016.

[19] Q. du, J. Liu, D. Tian et al., "Long noncoding RNA LINC00173 promotes NUTF2 expression through sponging miR-765 and facilitates tumorigenesis in glioma," Cancer Management and Research, vol. Volume 12, pp. 7211-7217, 2020.

[20] H. Zhang, "Rcircos: an R package for Circos 2D track plots," BMC Bioinformatics, vol. 14, no. 1, p. 244, 2013.

[21] A. Subramanian, P. Tamayo, V. K. Mootha et al., "Gene set enrichment analysis: a knowledge-based approach for interpreting genome-wide expression profiles," Proceedings of the National Academy of Sciences of the United States of America, vol. 102, no. 43, pp. 15545-15550, 2005.

[22] T. W. Li, J. Y. Fan, B. B. Wang et al., "Timer: a web server for comprehensive analysis of tumor-infiltrating immune cells," Cancer Research, vol. 77, no. 21, pp. E108-E110, 2017.

[23] T. W. Li, J. X. Fu, Z. X. Zeng et al., “Timer2.0 for analysis of tumor-infiltrating immune cells," Nucleic Acids Research, vol. 48, no. W1, pp. W509-W514, 2020.

[24] E. Becht, N. A. Giraldo, L. Lacroix et al., "Estimating the population abundance of tissue-infiltrating immune and stromal cell populations using gene expression," Genome Biology, vol. 17, no. 1, p. 249, 2016.

[25] A. Subramanian, R. Narayan, S. M. Corsello et al., "A next generation connectivity map: L1000 platform and the first 1,000,000 profiles," Cell, vol. 171, article e1417, pp. 14371452, 2017.

[26] G. Xiaoyu, Y. Yanpin, T. Yan, and H. Guangwu, "Expression and significance of nuclear transporter 2 in head and neck squamous cell carcinoma," Journal of Guangxi Medical University, vol. 37, pp. 177-181, 2020.

[27] B. Li, H. Q. Zhang, Y. Shi et al., "Overexpression of nuclear transport factor 2 may protect against diabetic retinopathy," Molecular Vision, vol. 15, pp. 861-869, 2009.

[28] L. G. Sheffield and H. B. Miskiewicz, "Nuclear pore complex proteins in Alzheimer disease," Journal of Neuropathology \& Experimental Neurology, vol. 65, no. 1, pp. 45-54, 2006.

[29] W. Hu, "Kinetic properties of nuclear transport conferred by the retinoblastoma (Rb) NLS," Journal of Cellular Biochemistry, vol. 95, no. 4, pp. 782-793, 2005.

[30] S. H. Park, "Reduction of exportin 6 activity leads to actin accumulation via failure of RanGTP restoration and NTF2 sequestration in the nuclei of senescent cells," Experimental Cell Research, vol. 317, no. 7, pp. 941-954, 2011.
[31] L. D. Vuković, K. H. White, J. P. Gigley, and D. L. Levy, "Nuclear transport factor 2 (NTF2) suppresses metastatic melanoma by modifying cell migration, metastasis, and gene expression," bioRxiv, 2020.

[32] L. D. Vukovic, B. A. Stohr, and D. L. Levy, "Abstract 4456: Increased ntf2 levels in melanoma cell lines affect nuclear size and cancer cell characteristics," Cancer Research, vol. 78, pp. 4456-4456, 2018.

[33] J. B. Swann and M. J. Smyth, "Immune surveillance of tumors," The Journal of Clinical Investigation, vol. 117, no. 5, pp. 11371146, 2007.

[34] T. F. Gajewski, "Innate and adaptive immune cells in the tumor microenvironment," Nature Immunology, vol. 14, no. 10, pp. 1014-1022, 2013.

[35] S. J. F. Cronin and J. M. Penninger, "From t-cell activation signals to signaling control of anti-cancer immunity," Immunological Reviews, vol. 220, no. 1, pp. 151-168, 2007.

[36] K. M. Fracchia, "Modulation of t cell metabolism and function through calcium signaling," Frontiers in Immunology, vol. 4, 2013.

[37] L. P. Chen and D. B. Flies, "Molecular mechanisms of T cell costimulation and co-inhibition," Nature Reviews. Immunology, vol. 13, no. 4, pp. 227-242, 2013.

[38] J. M. Dal Porto, S. B. Gauld, K. T. Merrell, and D. Mills, "B cell antigen receptor signaling 101," Molecular Immunology, vol. 41, no. 6-7, pp. 599-613, 2004.

[39] Y. Li, K. Wang, Y. Z. Jiang et al., "ITE suppresses growth of human ovarian cancer," Reproductive Sciences, vol. 18, pp. 145a-145a, 2011.

[40] L. K. Puntigam, S. S. Jeske, M. Götz et al., "Immune checkpoint expression on immune cells of HNSCC patients and modulation by chemo- and immunotherapy," International Journal of Molecular Sciences, vol. 21, no. 15, p. 5181, 2020.

[41] S. A. Piwarski, C. Thompson, A. R. Chaudhry et al., "The putative endogenous AHR ligand ITE reduces JAG1 and associated NOTCH1 signaling in triple negative breast cancer cells," Biochemical Pharmacology, vol. 174, article 113845, 2020.

[42] L. F. Nugent, G. P. Shi, B. P. Vistica, and O. Ogbeifun, "ITE, a novel endogenous nontoxic aryl hydrocarbon receptor ligand, efficiently suppresses EAU and T-cell-mediated immunity," Investigative Ophthalmology \& Visual Science, vol. 54, no. 12, pp. 7463-7469, 2013.

[43] J. D. Abron, N. P. Singh, M. K. Mishra et al., "An endogenous aryl hydrocarbon receptor ligand, ITE, induces regulatory $\mathrm{T}$ cells and ameliorates experimental colitis," American Journal of Physiology-Gastrointestinal and Liver Physiology, vol. 315, no. 2, pp. G220-G230, 2018.

[44] A. B. Miller, A. T. Loynachan, H. M. Bush et al., "Effects of pituitary pars intermedia dysfunction and prascend (pergolide tablets) treatment on endocrine and immune function in horses," Domestic Animal Endocrinology, vol. 74, article 106531, 2021.

[45] I. Kuss, B. Hathaway, and R. L. Ferris, "Decreased absolute counts of $\mathrm{T}$ lymphocyte subsets and their relation to disease in squamous cell carcinoma of the head and neck," Clinical Cancer Research: An Official Journal of the American Association for Cancer Research, vol. 10, no. 11, pp. 3755-3762, 2004. 